\title{
Primary small-bowel adult T-cell leukemia/ lymphoma with gastric AL amyloidosis
}

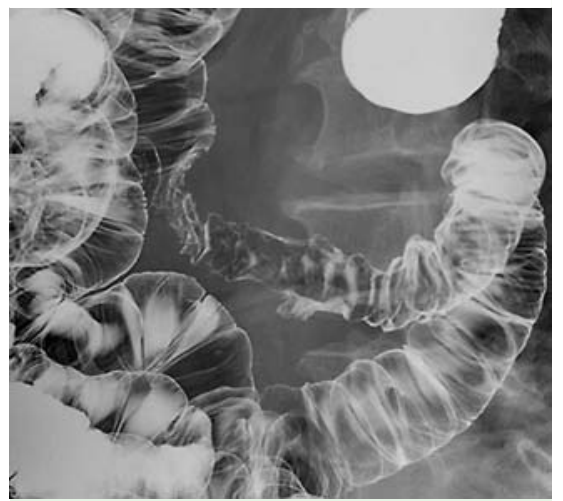

Fig. 1 Primary small-bowel adult T-cell leukemia/lymphoma with gastric AL amyloidosis. Small bowel enteroclysis demonstrates a narrowing edematous lesion with multiple ulcerations in the jejunum.

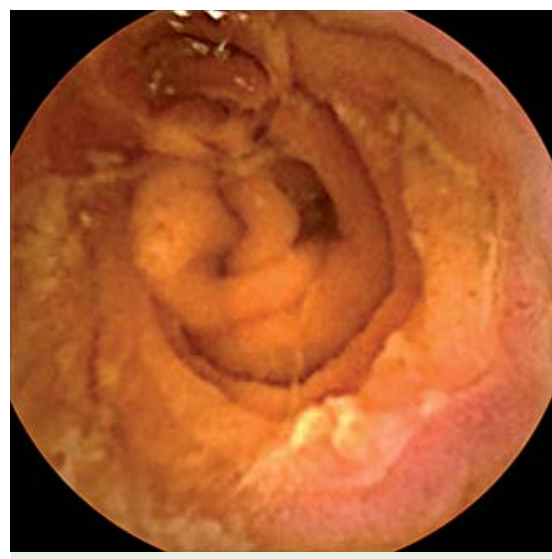

Fig. 2 Capsule endoscopy shows multiple ulcers with enlarged Kerckring folds in the jejunum.

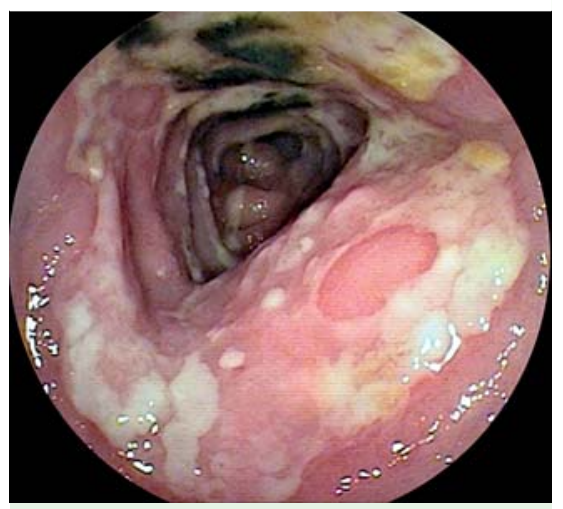

Fig. 3 Double-balloon endoscopy reveals enlarged Kerckring folds with annular and irregularly shaped shallow ulcers in the jejunum.
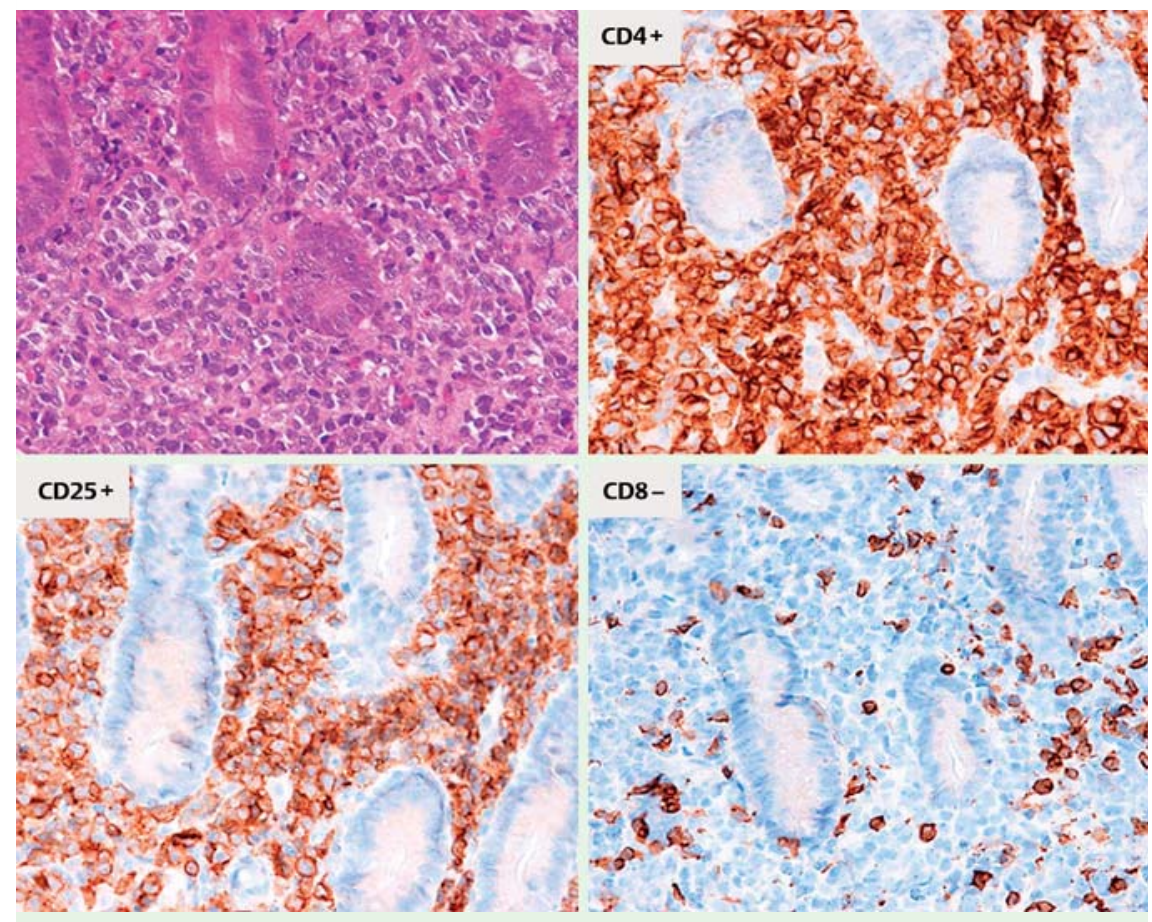

Fig. 4 Histology of a biopsy specimen from the jejunal ulcer showing diffuse infiltration of medium to large pleomorphic lymphoid cells with CD4+, CD25+, CD8 - immunophenotype (magnification $\times 400$ ).

An 86-year-old man was admitted because of small-bowel wall thickening and mesenteric lymphadenopathy. Neither peripheral lymphadenopathy nor hepatosplenomegaly was noted. The leukocyte count was $7930 / \mathrm{mm}^{3}$, and no abnormal lymphocytes were found. The result of testing for serum antihuman T-lymphotropic virus type 1 (HTLV-1) antibody was positive. A bone marrow biopsy was normal without evidence of lymphoid infiltration. Smallbowel enteroclysis demonstrated a narrowing, edematous lesion with multiple ulcerations in the jejunum ( $\bullet$ Fig. 1 ). Capsule endoscopy ( $\bullet$ Fig. 2 ) and doubleballoon endoscopy ( $\bullet$ Fig.3) showed enlarged Kerckring folds with annular and irregularly shaped shallow ulcers. Biopsy specimens from the ulcers revealed diffuse infiltration of medium to large pleomorphic lymphoid cells with CD3+, CD4+, CD5+, CD25+, CCR4+, FoxP3+, CD8-, CD20-, CD56-, and TIA1 - immunophenotype ( $\odot$ Fig. 4). Biopsy specimens from the stomach, duodenum, ileum, and colon showed no lymphoma cells. Fluorodeoxyglucose F 18 positron emission tomography showed abnormal uptake in the jejunal mass, wide areas of mesentery, and para-aortic lymph nodes. Esophagogastroduodenoscopy with chromoendoscopy revealed granular stomach mucosa and swollen folds in the corpus, with panatrophic gastritis ( $\bullet$ Fig. 5 ). Biopsy from the granular mucosa and folds showed massive amyloid deposits of homogeneous acidophilic substances in the submucosa and around the muscularis mucosae ( $\bullet$ Fig. 6 ), which were immunohistochemically positive for $\lambda$ light chain. Amyloid deposits were not observed in any specimen taken from the duodenum, jejunum, ileum, and colon. Therefore, the patient's diagnosis was stage IIE (Lugano classification) small-bowel adult T-cell leukemia/lymphoma (ATLL) accompanied by gastric AL amyloidosis.

Although systemic ATLL often involves the small bowel [1-3], primary small-bowel ATLL is extremely rare $[4,5]$. Endoscopic findings of small-bowel involvement with ATLL have included multiple polypoid or reddish elevated lesions $[2,3]$. However, we believe that the enlarged Kerckring folds with annular or irregularly shaped shallow ulcers observed in our case may be characteristic in primary small-bowel ATLL. 


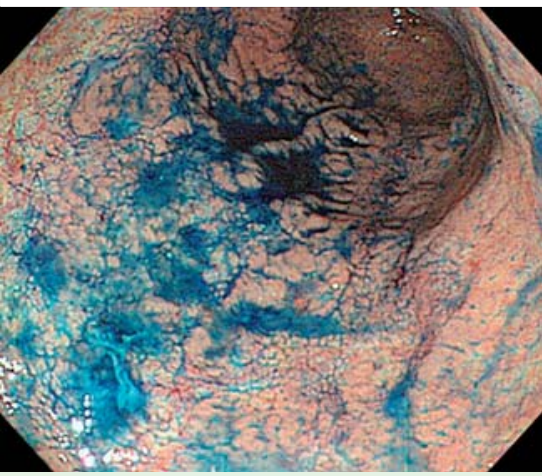

Fig. 5 Esophagogastroduodenoscopy reveals granular mucosa and swollen folds in the corpus, with pan-atrophic gastritis.

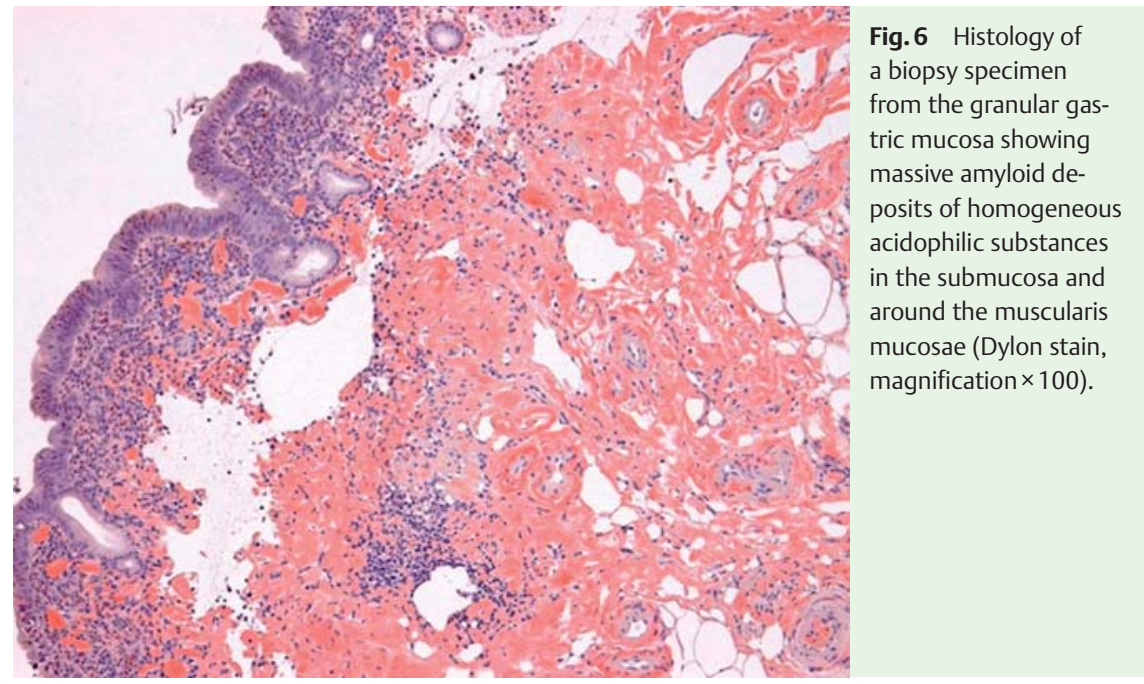

\section{References}

1 Utsunomiya A, Hanada S, Terada $A$ et al. Adult T-cell leukemia with leukemia cell infiltration into the gastrointestinal tract. Cancer 1988; 61: 824-828

2 Itsuno M, Makiyama M, Muta $\mathrm{K}$ et al. Adult T-cell leukemia with multiple lymphomatous polyposis of the gastrointestinal tract. Endoscopy 1995; 27: 700 - 703

3 Isomoto $\mathrm{H}$, Ohnita $\mathrm{K}$, Haraguchi $\mathrm{M}$ et al. Jejunal perforation in a patient with adult T-cell leukemia. Leuk Lymphoma 2001; 42: 1423 1427

4 Cabrera ME, Labra S, Ford A et al. HTLV-1 induced intestinal lymphoma. Leuk Lymphoma 1999; 35: 637-640

5 Katoh A, Ohshima K, Kanda M et al. Gastrointestinal $\mathrm{T}$ cell lymphoma: predominant cytotoxic phenotypes, including alpha/beta, gamma/delta T cell and natural killer cells. Leuk Lymphoma 2000; 39: 97-111

\section{Bibliography}

DOI http://dx.doi.org/ 10.1055/s-0034-1390757

Endoscopy 2014; 46: E613-E614

(c) Georg Thieme Verlag KG

Stuttgart · New York

ISSN 0013-726X

\section{Corresponding author}

\section{Hiroki Yaita, MD}

Division of Gastroenterology

Matsuyama Red Cross Hospital

1 Bunkyo-cho, Matsuyama-shi

Ehime 790-8524

Japan

Fax: +81-89-926-9916

hyaita@matsuyama.jrc.or.jp 